\title{
Ultrasound mapping of new cannulation sites for arteriovenous fistulas/grafts at South Tyneside and Sunderland NHS Foundation Trust (STSFT) Renal Unit.
}

\author{
Haroon Ayub ${ }^{1,2}$, Rauri Clark ${ }^{1}$, Shalabh Srivastava ${ }^{1,2}$, James Andrews ${ }^{1}$, Debra Sweeney ${ }^{1}$, Saeed Ahmed ${ }^{1}$ \\ ${ }^{1}$ Department of Renal Medicine, South Tyneside and Sunderland NHS Foundation Trust, UK \\ ${ }^{2}$ Institute of Genetic Medicine, Newcastle University, UK
}

\begin{abstract}
:
Vascular access remains the lifeline for dialysis population where arteriovenous fistula/graft cannulation plays a pivotal role in the well-being of these patients. The cannulation specially becomes difficult in elderly, diabetic and obese patients. The accessibility of an AV fistula largely relies on the condition of the patient's veins, which often leads to difficulty in cannulation with the traditional blind method. South Tyneside and Sunderland NHS Foundation Trust (STSFT) Renal unit adopted ultrasound mapping of new cannulation sites for arteriovenous fistulas and arteriovenous grafts. The aim of this study was to evaluate the qualitative impact of this practice on the dialysis patients.
\end{abstract}

This was a qualitative study and was conducted in the Renal Unit at STSFT. Haemodialysis patients with deep or small vessels for cannulation, a vessel with a history of frequent multiple cannulation failures and extravasations and a vessel whose cannulation on the first attempt was vital were included in the study. AVF/AVG were mapped using Doppler ultrasound (US) undertaken by a consultant Interventional Nephrologist (IN). The map of the AVF/AVG and new cannulation sites were captured as an image on the patients' smart phone to act as record to show the dialysis nurse cannulating the AVF/AVG. It was hoped that this would encourage rope laddering and AVF/AVG preservation.

Patients reported no significant improvement in cannulation process even after US mapping of new cannulation sites. Patients did report a better understanding of the AVF/AVG geometry especially amongst the self cannulators on home haemodialysis. According to the patients the successful cannulation was mainly dependent on the skills of dialysis nurses rather than the US mapping of the AVF/AVG being of any assistance.

Key Words: Arteriovenous Fistula, hemodialysis, rope ladder, buttonhole, cannulation. Corresponding Author:

Dr. Haroon Ayub

Specialty Registrar (ST7)

Department of Renal Medicine

South Tyneside and Sunderland NHS Foundation Trust

Associate Clinical Researcher

Institute of Genetic Medicine

Newcastle University

Newcastle Upon Tyne, UK

Email: haroonayub177@gmail.com

PJKD 2020;(11):199-202

\section{Introduction}

Vascular access remains the lifeline for dialysis population where arteriovenous fistula/graft cannulation plays a pivotal role in the well-being of these patients. ${ }^{1}$ The cannulation specially becomes difficult in elderly, diabetic and obese patients ${ }^{2}$. The accessibility of an AV fistula largely relies on the condition of the patient's veins, which often leads to difficulty in

cannulation with the traditional blind method. ${ }^{3}$ South Tyneside and Sunderland NHS Foundation Trust (STSFT) Renal unit adopted ultrasound mapping of new cannulation sites for arteriovenous fistulas and arteriovenous grafts. ${ }^{4}$ The aim of this study was to evaluate the qualitative impact of this practice on the dialysis patients.

The complex nature of the patients who require permanent vascular access makes it essential that a comprehensive, multidisciplinary approach be adopted. ${ }^{5}$ The creation and maintenance of functioning access and the associated complications are responsible for significant morbidity, 
hospitalization, and cost for patients with end-stage renal disease. Good judgment for access-site selection, technical surgical excellence, and appropriate management of complications are the primary objectives for long-term success. However, other factors, such as regular vascular access surveillance postoperatively and central coordination by a dedicated access coordinator for seamless communication among nephrologists, surgeons, and radiologists, are equally important in ensuring a practical and cost-effective access program. ${ }^{6,7}$

Vascular arteriovenous fistulae (AVF) -related complications remain an important cause of morbidity and health care usage in haemodialysis service provision. AVFs are the preferred access modality and associated with the lowest rates of access-related events. ${ }^{8,9}$

Cannulation of AVF is by the traditional rope ladder (RL) also known as sharp needle) technique, using venepuncture with sharp needles at a new site during each dialysis session, or the buttonhole ( $\mathrm{BH}$; also known as blunt needle) technique, involving repeated punctures with blunt needles through established tissue tunnel tracts. The BH technique was initially introduced with hopes of preserving access in short AVFs. ${ }^{10}$ The perceived benefits of reduction in problematic cannulation episodes and patient discomfort and lower requirement for AVF interventions have led to a resurgence in the use of BH cannulation, specifically in the home haemodialysis (HHD) setting.

Recent studies have suggested increased infections and aneurysmal formation in BH cannulation. Studies need to be done to evaluate the long-term complications in patients with RL cannulation under Doppler ultrasound (US) guidance.

\section{Materials and methods}

This was a prospective qualitative study and was conducted in the Renal Unit at South Tyneside and Sunderland NHS Foundation Trust (STSFT) from June 2019 to August 2019. 38 haemodialysis patients fulfilling the inclusion criteria had vein mapping of AVF/AVG by a consultant Interventional Nephrologist (IN) for new cannulation sites, using Mindray TE7 ultrasound system (Shenzhen, China) having L14-6ns Linear Array Probe used with frequency range of $\mathbf{6 - 1 4 M H z}$. The map of the AVF/AVG and new cannulation sites were captured as an image on the patients' smart phone to act as record to show the dialysis nurse cannulating the AVF/AVG. It was hoped that this would encourage rope laddering and AVF/AVG preservation. Patients having history of frequent multiple cannulation failures and extravasations a vessel whose cannulation on the first attempt was vital were included. Patients were followed to evaluate improvement in cannulation techniques after the AVF/AVG scanning. Outcome was simply assessed by patients' satisfaction after the use of US assisted vein mapping.

- Patients having vascular access problems

- Referred to vascular access nurse

- Booked to be seen by interventional nephrologist

- US mapping done

- Pictures taken on patient's mobile phone

- New cannulation sites used by dialysis nurses/patients using the picture on mobile phone

\section{Results}

There was no improvement reported by most of the patients in cannulation process even after US mapping of new cannulation sites. Patients did report a better understanding of the AVG/AVG geometry especially amongst the self cannulators on home haemodialaysis, however these patients were still more comfortable with buttonhole technique of AVF cannulation. According 
to the patients the successful cannulation was mainly dependent on the skills of dialysis nurses rather than the US mapping of the AVF/AVG being of any assistance.

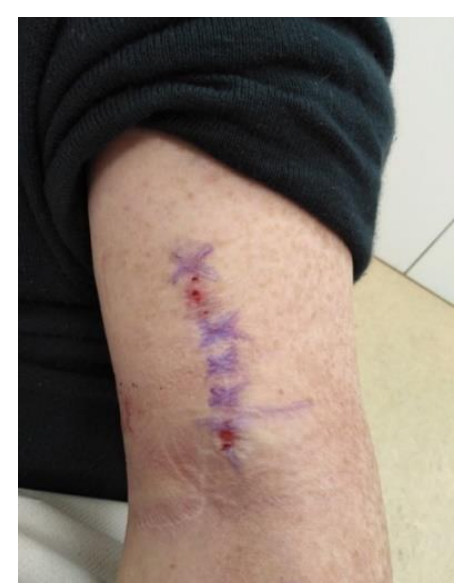

Figure 1: Image shows new marking sites for AVF cannulation in brachiocephalic AVF

Out of 38 patients, $31(81.5 \%)$ patients reported no improvement in cannulation techniques after using US for mapping cannulation points. 7 (18.5\%) patients however did report some improvement, which they attributed to better understanding of AVF/AVG anatomy by the healthcare providers at the dialysis unit.

\section{Discussion}

The results were contrary to what were expected by the authors. It was hypothesized that AVF/AVG mapping would help the dialysis nurses to cannulate the patients easily and would be more comfortable for the patients as well. Blind cannulation in difficult to cannulate AVF/AVG can lead to bruising and hematoma formation. ${ }^{11}$ This in turn results in a vicious cycle with further difficult cannulation. However, it turned out that skills and expertise of dialysis nurses seemed more important in cannulation procedures than the AVF/AVG markings.

Experienced dialysis nurses seem to overcome the hurdles of difficult cannulation even in complex ACF/AVG. These experienced nurses are hard to find in any dialysis centre, so the focus should be make cannulation easy for less experienced nurses as well. Moreover, rope ladder technique should be encouraged to prevent complications like infections and aneurysm formation. ${ }^{12,13}$ Buttonhole technique although associated with less hematoma formation and fewer cannulation problems does not confer increased life for the access. ${ }^{14}$ It is still a matter of debate whether buttonhole or rope ladder technique is superior for access patency. However, the authors believe that if AVF/AVG is cannulated through rope ladder technique, it would not only help to utilize the whole venous segment but also prevent complications like cellulitis and aneurysm formation. Having said that, expertise is required both on the part of nephrologist and dialysis nurses to commit to this practise. Nurse education on rope laddering is extremely important as it is a feared practise in many.

Real time US by dialysis nursing staff for cannulating AVG/AVF will help in these difficult cannulations and would be a regular practise in the near future. Newer dialysis machines should come with an attached US probe. In this manner AVF/AVG can be scanned by the dialysis nurses at bedside prior to cannulation. This would also increase interest and motivation amongst dialysis nurses to learn new cannulation techniques. 
Randomised prospective trial needs to be done with larger cohort of patients to analyse this hypothesis. Rope ladder technique might not be comfortable for the patient but would prevent certain complications if done under US guidance.

\section{Conflict of Interest: None Declared}

\section{References}

1. Pisoni RL, Zepel L, Fluck R, Lok CE, Kawanishi H, Süleymanlar G, Wasse H, Tentori F, Zee J, Li Y, Schaubel D. International differences in the location and use of arteriovenous accesses created for hemodialysis: results from the Dialysis Outcomes and Practice Patterns Study (DOPPS). Am J Kidney Diseases. 2018;71(4):469-78.

2. Beathard GA, Lok CE, Glickman MH, Al-Jaishi AA, Bednarski D, Cull DL, Lawson JH, Lee TC, Niyyar VD, Syracuse D, Trerotola SO. Definitions and end points for interventional studies for arteriovenous dialysis access. Clin J Am Soc Nephrol 2018 Mar 7;13(3):501-12.

3. Parisotto MT. Cannulation Technique Influences Arteriovenous Fistula and Graft Survival. Nephrol Nurs J. 2018 Mar 1;45(2).

4. Coventry LL, Hosking JM, Chan DT, Coral E, Lim WH, Towell-Barnard A, Twigg DE, Rickard CM. Variables associated with successful vascular access cannulation in hemodialysis patients: a prospective cohort study. BMC Nephrol. 2019 Dec;20(1):197.

5. Allon M, Bailey R, Ballard R, Deierhoi MH, Hamrick K, Oser R, Rhynes VK, Robbin ML, Saddekni S, Zeigler ST. A multidisciplinary approach to hemodialysis access: prospective evaluation. Kidney Int. 1998;53(2):473-9.

6. Bay WH, Henry ML, Lazarus JM, Lew NL, Ling J, Lowrie EG. Predicting hemodialysis access failure with color flow Doppler ultrasound. Am J Nephrol. 1998;18(4):296-304.

7. Lin SL, Chen HS, Huang CH, Yen TS. Predicting the outcome of hemodialysis arteriovenous fistulae using duplex ultrasonography. J Formosan Med Assoc Taiwan yi zhi. 1997;96(11):864-8.

8. Patel AA, Tuite CM, Trerotola SO. K/DOQI Guidelines: What Should an Interventionalist Know? In Seminars in interventional radiology 2004 Jun (Vol. 21, No. 02, pp. 119-124). Copyright $\odot 2004$ by Thieme Medical Publishers, Inc., 333 Seventh Avenue, New York, NY 10001, USA..

9. Taylor G, Gravel D, Johnston L, Embil J, Holton D, Paton S, Canadian Nosocomial Infection Surveillance Program, Canadian Hospital Epidemiology Committee. Incidence of bloodstream infection in multicenter inception cohorts of hemodialysis patients. Am J Infec Control. 2004;32(3):155-60.

10. van Loon MM, Goovaerts T, Kessels AG, van der Sande FM, Tordoir JH. Buttonhole needling of haemodialysis arteriovenous fistulae results in less complications and interventions compared to the ropeladder technique. Nephrol Dial Transplant. 2009;25(1):225-30.

11. Verhallen AM, Kooistra MP, van Jaarsveld BC. Cannulating in haemodialysis: rope-ladder or buttonhole technique?. Nephrol DIal Transplant. 2007;22(9):2601-4.

12. Wong B, Muneer M, Wiebe N, Storie D, Shurraw S, Pannu N, Klarenbach S, Grudzinski A, Nesrallah G, Pauly RP. Buttonhole versus rope-ladder cannulation of arteriovenous fistulas for hemodialysis: a systematic review. Am J Kidney Dis. 2014;64(6):918-36.

13. MacRae JM, Ahmed SB, Atkar R, Hemmelgarn BR. A randomized trial comparing buttonhole with rope ladder needling in conventional hemodialysis patients. Clin J Am Soc Nephrol. 2012;7(10):1632-8.

14. Chan MR, Shobande O, Vats H, Wakeen M, Meyer X, Bellingham J, Astor BC, Yevzlin AS. The Effect of Buttonhole Cannulation vs. Rope- ladder Technique on Hemodialysis Access Patency. In Seminars in dialysis 2014 Mar (Vol. 27, No. 2, pp. 210-216). 